\title{
Comparison of the Fermentation Activities and Volatile Flavor Profiles of Chinese Rice Wine Fermented Using an Artificial Starter, a Traditional JIUYAO and a Commercial Starter
}

\author{
Chen Chen, Zheng Liu, Wenya Zhou, Huaixiang Tian, Juan Huang, Haibin Yuan and \\ Haiyan $Y^{*}$ *
}

Department of Food Science and Technology, Shanghai Institute of Technology, Shanghai, China

\section{OPEN ACCESS}

Edited by:

Xucong LV,

Fuzhou University, China

Reviewed by:

Yongjun Xia,

University of Shanghai for Science and Technology, China

Sheng Yin,

Beijing Technology and Business

University, China

*Correspondence:

Haiyan Yu

hyyu@sit.edu.cn

Specialty section:

This article was submitted to

Food Microbiology,

a section of the journal

Frontiers in Microbiology

Received: 28 May 2021

Accepted: 24 August 2021 Published: 20 September 2021

Citation:

Chen C, Liu Z, Zhou W, Tian H, Huang J, Yuan H and Yu H (2021) Comparison of the Fermentation Activities and Volatile Flavor Profiles of Chinese Rice Wine Fermented Using an Artificial Starter, a Traditional JIUYAO and a Commercial Starter.

Front. Microbiol. 12:716281. doi: 10.3389/fmicb.2021.716281
In this study, an artificial starter culture was prepared using the core microbial species of JIUYAO to produce Chinese rice wine (CRW). The fermentation activity and flavor profiles of CRW samples fermented with traditional JIUYAO, a commercial starter culture, and our artificial starter culture were compared. The optimal protectant combination for lyophilization of the artificial starter was established as $15.09 \%$ skim milk, $4.45 \%$ polyethylene glycol, 1.96\% sodium glutamate, and $11.81 \%$ maltodextrin. A comparative analysis revealed that the ethanol content of the three CRW samples was similar. The total acid content of the CRW sample fermented with the artificial starter $(7.10 \mathrm{~g} / \mathrm{L})$ was close to that of the sample fermented with JIUYAO (7.35 g/L), but higher than that of the sample fermented with the commercial starter (5.40 g/L). An electronic nose analysis revealed that the olfactory fingerprints of the CRW samples fermented with JIUYAO and the artificial starter resembled each other. For both above mentioned samples, the flavor profiles determined by gas chromatography-mass spectrometry indicated some differences in the variety and content of the aroma compounds, but the key odorants (odor activity values $\geq 1$ ), such as isoamyl acetate, ethyl acetate, phenyl alcohol, and isoamyl alcohol, were similar.

Keywords: Chinese rice wine, artificial starter, JIUYAO, flavor profiles, response surface methodology

\section{INTRODUCTION}

Chinese rice wine (CRW), which has a high nutritional value and distinctive flavor, has been consumed for centuries (Xu et al., 2015; Huang Z. R. et al., 2018). CRW includes many renowned types, such as Shaoxing, Jimo, and Fujian rice wine (Jiao et al., 2017). The best-known CRW, Shaoxing rice wine, is generally produced using glutinous rice, traditional JIUYAO, and wheat $q u$ (Chen et al., 2013). JIUYAO is a mixed starter culture that mainly includes bacteria, molds, and yeast and is responsible for the starch saccharification and fermentation in Shaoxing rice wine brewing (Liu et al., 2018). The microorganisms in JIUYAO are believed to play a crucial role in the fermentation activity and unique flavor formation of Shaoxing rice wine (Xie et al., 2007). 
Zhao et al. (2020) revealed that Proteobacteria and Firmicutes are the dominant bacterial phyla in JIUYAO. Our previous study (Chen et al., 2020) found that only five core species, two from the Weissella genus and one each from the Pediococcus, Saccharomycopsis, and Rhizopus genera, play a key role in the flavor formation and fermentation activity of CRW.

Currently, commercial starters that mainly include yeast and Rhizopus are widely used in the industrial brewing of CRW (Ferrer-Gallego et al., 2014). Although the fermentation period of CRW brewed with commercial starters is comparable to that of CRW brewed with traditional JIUYAO (Huang et al., 2019), the flavor of the former is inferior due to the limited microbial variety in the commercial starters (Liu et al., 2019). However, as traditional JIUYAO is usually handcrafted, its limitation is the instability of the resulting CRW's quality and flavor profiles across batches (Zhao and Zhang, 2010). Therefore, it would be of great significance to develop an artificial starter culture as a replacement for traditional JIUYAO that could yield CRW batches of high quality and uniform flavor.

A microbial consortium selected as a starter culture should be able to withstand subsequent fermentation. Therefore, lyophilization techniques and low temperatures (refrigeration or freezing) are usually used to stabilize the starter culture (Abadias et al., 2001; Pradelles et al., 2009). Lyophilization is a simple technique to maintain a high number of viable microorganisms in a starter culture inoculum in powder form, as it involves few procedural steps. However, this preservation process can cause cell damage or lead to cell death (Ale et al., 2015). Cell mortality during lyophilization can be minimized by optimizing freeze-drying conditions and using lyoprotectants (Cerrutti et al., 2000; Hubálek, 2003), such as proteins, skim milk, sugars, and other biopolymers (Coulibaly et al., 2016). Although freeze-dried bacterial powder with protectants has been used as a starter culture (Chen H. et al., 2015), its use in place of traditional JIUYAO for CRW brewing has not been documented.

In this study, an artificial starter culture was prepared by combining the core microorganisms from traditional JIUYAO that contribute to the unique flavor of CRW, and then freezedrying them to powder form using a lyoprotectant combination optimized through a response surface optimization experiment. The fermentation activity and flavor profile of the CRW samples fermented with this artificial starter were compared with those of samples fermented with traditional JIUYAO and a commercial starter culture. The use of such an artificial starter can improve and standardize the fermentation activity and aroma quality

TABLE 1 | Experimental factor levels of the Box-Behnken design.

\begin{tabular}{lccc}
\hline Factors & \multicolumn{3}{c}{ Level } \\
\cline { 2 - 4 } & $\mathbf{- 1}$ & $\mathbf{0}$ & $\mathbf{1}$ \\
\hline A skim milk (\%) & 10 & 15 & 20 \\
B polyethylene glycol (\%) & 3 & 5 & 7 \\
C sodium glutamate (\%) & 1.5 & 2 & 2.5 \\
D maltodextrin (\%) & 5 & 10 & 15
\end{tabular}

of CRW, and further promote the industrial production of traditional CRW.

\section{MATERIALS AND METHODS}

\section{JIUYAO, Starter Culture and Lyoprotectants}

JIUYAO samples were obtained from Zhejiang Tapai Shaoxing Rice Wine Co., Ltd., Shaoxing city, Zhejiang province, China. The samples were refrigerated at $4^{\circ} \mathrm{C}$ before being transported to the laboratory. A commercial starter culture composed of Saccharomyces cerevisiae and Rhizopus oryzae was purchased from a commercial yeast and yeast extract manufacturer in China. Five microbial species, namely Pediococcus pentosaceus (CCTCC M 2019323), Weissella cibaria (BNCC206838), W. confusa (CICC23465), Saccharomycopsis fibuligera (CCTCC M 2019324), and $R$. arrhizus (CCTCC M 2019325), were selected and isolated as the core functional microbes from the JIUYAO samples, due to their key role in the fermentation activity and flavor formation of CRW, as determined in our previous study (Chen et al., 2020). To ensure that the samples had the ability to produce ethanol, S. cerevisiae (NKCCMR NK3. 00156) was also added to the artificial starter (Chen et al., 2020). The isolated strains were stored at $-80^{\circ} \mathrm{C}$ and used after reactivation by successive subcultures in steamed rice. Briefly, for activation, $W$. cibaria, $W$. confusa, and P. pentosaceus $(2 \%$ vol/vol) were inoculated into MRS broth (Merck, Darmstadt, Germany) and incubated at $37^{\circ} \mathrm{C}$ for $18 \mathrm{~h}$, whereas $S$. fibuligera, $S$. cerevisiae, and $R$. arrhizus ( $2 \% \mathrm{vol} / \mathrm{vol})$ were inoculated in MEB liquid medium (Merck, Darmstadt, Germany) and incubated at $30^{\circ} \mathrm{C}$ for $24 \mathrm{~h}$. The inoculum concentrations of the individual core bacterial species were determined according to the amount of JIUYAO added to the CRW, the total number of bacterial and fungal colonies in the JIUYAO, and the abundance of core microbial species in JIUYAO as described in our previous study (Chen et al., 2020). The final concentrations of the core microbes that were combined to form the artificial starter culture were $P$. pentosaceus $\left(8.6 \times 10^{3} \mathrm{CFU} / \mathrm{g}\right)$, S. fibuligera $\left(9.6 \times 10^{3} \mathrm{CFU} / \mathrm{g}\right), R$. arrhizus $\left(10.6 \times 10^{2} \mathrm{CFU} / \mathrm{g}\right), W$. cibaria $\left(2.2 \times 10^{3} \mathrm{CFU} / \mathrm{g}\right), W$. confuse $\left(2.2 \times 10^{3} \mathrm{CFU} / \mathrm{g}\right)$, and S. cerevisiae $\left(2.0 \times 10^{4} \mathrm{CFU} / \mathrm{g}\right)$.

For use as lyoprotectants, skim milk was purchased from Fonterra Ltd. (New Zealand), and polyethylene glycol, sodium glutamate, and maltodextrin of food grade were purchased from WanBang Co. Ltd. (Zhengzhou, Henan Province, China).

\section{Freeze-Drying of the Starter}

All of the protectants used in the experiment were dissolved in distilled water to obtain various concentrations. They were sterilized at $115^{\circ} \mathrm{C}$ for $15 \mathrm{~min}$ and stored at $4^{\circ} \mathrm{C}$ until use.

Different concentrations of the protectant solutions were added to the culture pellets of the core microbes in the ratio 1:1 (vol/vol). After mixing, the samples were pre-frozen at $-80^{\circ} \mathrm{C}$ for $2 \mathrm{~h}$, placed in a vacuum freeze-dryer at $-80^{\circ} \mathrm{C}$ and 0.162 mbar vacuum for $48 \mathrm{~h}$, and finally stored at $-20^{\circ} \mathrm{C}$ until use (Qi et al., 2017). 
A

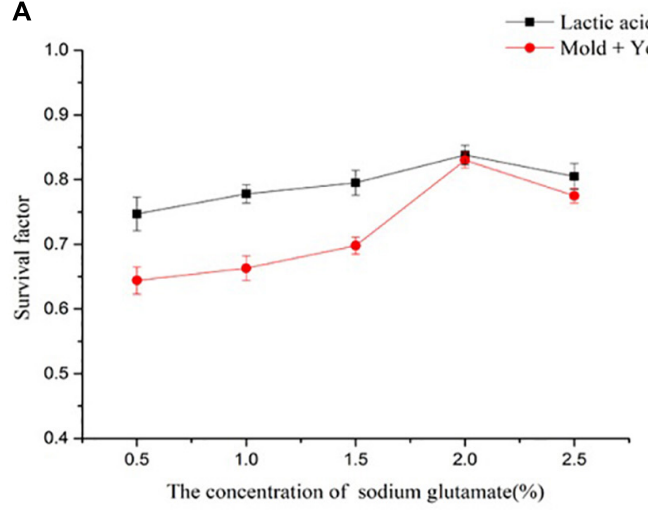

C

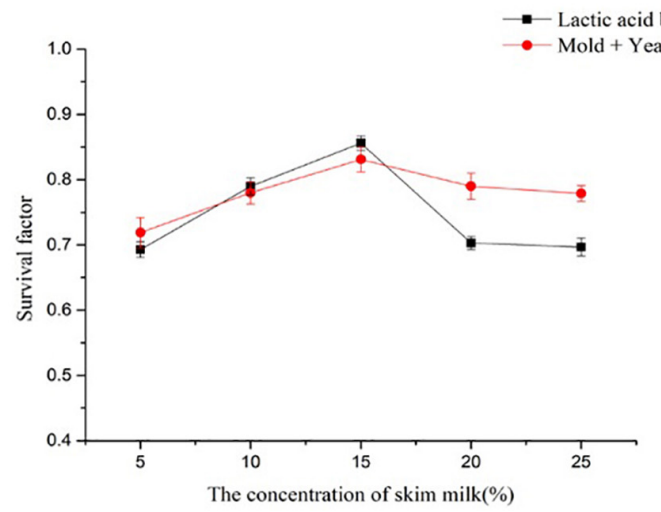

B

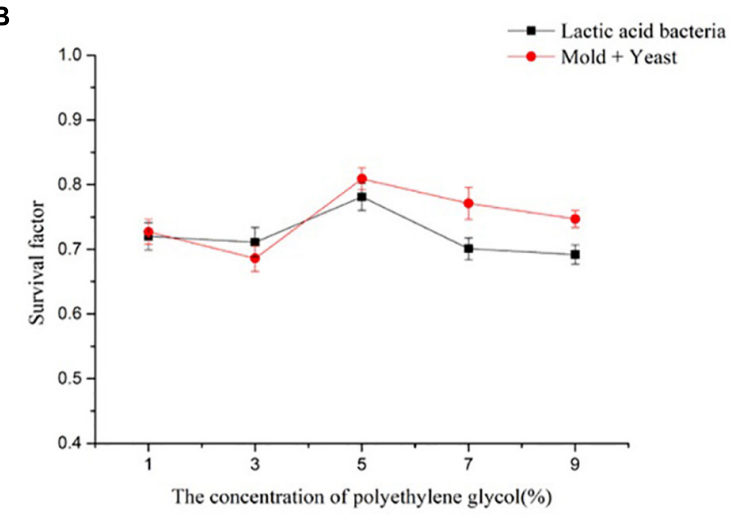

D

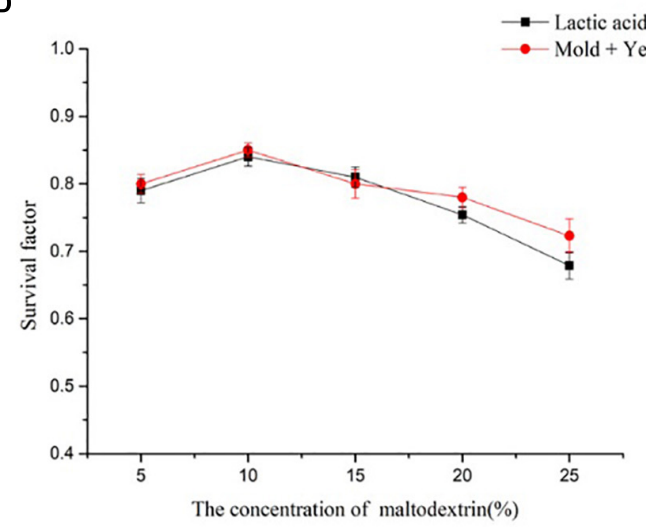

FIGURE 1 | Effects of different concentrations of four lyoprotectants on the survival factor of the artificial starter culture. (A) Sodium glutamate, (B) polyethylene glycol, (C) skim milk, and (D) maltodextrin.

TABLE 2 | Regression model analysis of variance.

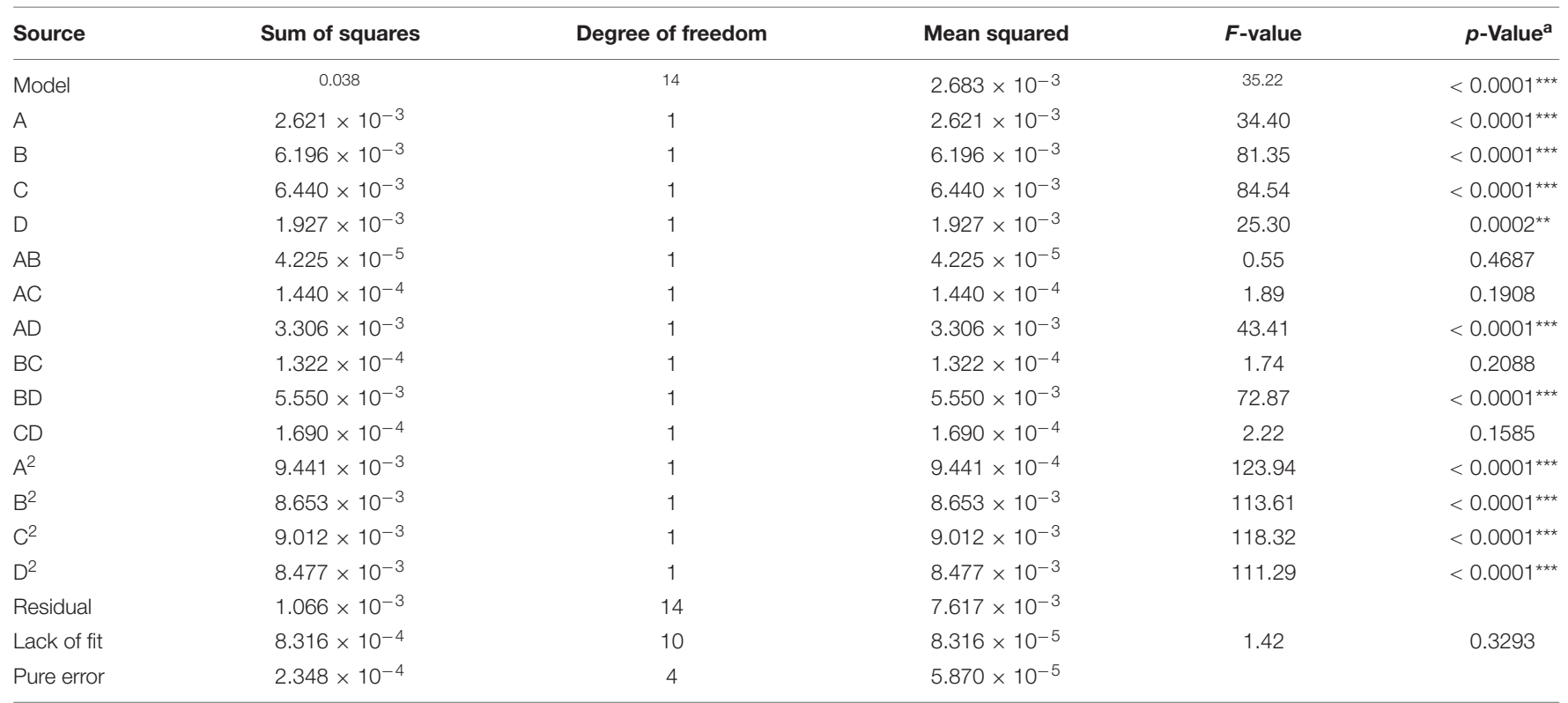

${ }^{a * * *} p<0.001$, extremely significant; ${ }^{* *} p<0.01$, highly significant; ${ }^{*} p<0.05$, significant. 
The lyophilization survival factor $\left(S F_{L}\right)$ was calculated following the formula given by Hubálek (2003):

$$
\begin{array}{r}
S F_{L}=1-\left[\left(\log C F U / m L_{\text {initial }}-\log C F U / m L_{\text {final }}\right) /\right. \\
\left.\log C F U / m L_{\text {initial }}\right]
\end{array}
$$

where $\mathrm{CFU} / \mathrm{mL}_{\text {initial }}$ is the number of viable cells before lyophilization, and $\mathrm{CFU} / \mathrm{mL}_{\text {final }}$ is the number of viable cells after lyophilization.

\section{Experimental Design for Optimization of the Lyoprotectant Composition}

A lyoprotectant is usually added to the target solution of cells before the freeze-drying process. It adds a matrix around the cells that protects them from drying and freezing and increases their survival ability (Abadias et al., 2001). Ten lyoprotectants were selected and mixed thoroughly with the core microbes, and the survival factors of the microbes were determined as an indicator of their survival in our preliminary study (data not shown). Finally, four lyoprotectants with the best protective effects were selected for our experiment: skim milk, polyethylene glycol, sodium glutamate, and maltodextrin. These selected lyoprotectants were diluted to different concentration gradients (skim milk and maltodextrin: 5, 10, 15, 20 and 25\%; polyethylene glycol: 1,3, 5, 7 and 9\%; sodium gluconate: $0.5,1,1.5,2$ and $2.5 \%$ ), and their optimal concentrations were determined based on the cell survival factor.

The lyoprotectant composition was further optimized using a four-factor, three-level Box-Behnken design and three levels of the $N=27$ test with Y (lyophilization survival rate) as the response value. The factor levels are shown in Table 1. The following polynomial equation was used:

$$
\begin{array}{r}
Y=a_{0}+a_{1} A+a_{2} B+a_{3} C+a_{4} D+a_{12} A B+a_{13} A C+a_{14} A D \\
+a_{23} B C+a_{24} B D+a_{34} C D+a_{11} A^{2}+a_{22} B^{2}+a_{33} C^{2}+a_{44} D^{2}
\end{array}
$$

where $\mathrm{Y}$ is the predicted response; $\mathrm{A}, \mathrm{B}, \mathrm{C}$, and $\mathrm{D}$ are independent variables representing the concentration of the four protectants skim milk, polyethylene glycol, sodium glutamate, and maltodextrin, respectively; $\mathrm{a}_{0}$ is the second-order reaction constant; $\mathrm{a}_{1}, \mathrm{a}_{2}, \mathrm{a}_{3}$, and $\mathrm{a}_{4}$ are the linear coefficients; $\mathrm{a}_{11}, \mathrm{a}_{22}, \mathrm{a}_{33}$, and $\mathrm{a}_{44}$ are the quadratic coefficients; and $\mathrm{a}_{12}, \mathrm{a}_{13}, \mathrm{a}_{14}, \mathrm{a}_{23}, \mathrm{a}_{24}$, and $\mathrm{a}_{34}$ are the interaction coefficients (Crowell and Ough, 1979).

\section{CRW Brewing}

The main steps in CRW brewing are rice soaking, steaming, cooling, starter addition, and fermentation. Briefly, glutinous rice $(100 \mathrm{~g})$ was soaked in $100 \mathrm{~mL}$ water for $12 \mathrm{~h}$ at $25^{\circ} \mathrm{C}$. After steaming the soaked rice for $20 \mathrm{~min}$ and then cooling it to 25$30^{\circ} \mathrm{C}$, rice fermentation was initiated by adding JIUYAO, the artificial starter, or the commercial starter at a final concentration of $0.002 \mathrm{~g} / \mathrm{g}$ steamed rice. The fermentation process was performed at $29^{\circ} \mathrm{C}$ for $30 \mathrm{~h}$. The acid content, ethanol content, and saccharification power were determined as described in our previous study (Chen et al., 2020). All chemical determination experiments were performed in triplicate.

\section{Flash GC Electronic Nose Detection}

A HERACLES flash GC electronic nose (Alpha M.O.S., Toulouse, France) equipped with an MXT-5 column and an MXT1701 column was used for the aroma analysis of the CRW samples. This instrument can perform a complete data analysis owing to its integration with classical gas chromatography (GC) functionalities and electronic nose (e-nose) olfactory fingerprint software.

Briefly, $5 \mathrm{~mL}$ of each CRW sample was added to a separate $20-\mathrm{mL}$ vial and incubated at $25^{\circ} \mathrm{C}$ for $30 \mathrm{~min}$. Hydrogen was circulated at a constant flow rate of $1 \mathrm{~mL} / \mathrm{min}$, and $5 \mathrm{~mL}$ of headspace gas was injected into the GC port at $200^{\circ} \mathrm{C}$. The temperature changes in the GC column were as follows: $50^{\circ} \mathrm{C}$ for $2 \mathrm{~s}$, a $1^{\circ} \mathrm{C} / \mathrm{s} \mathrm{ramp}$ to $80^{\circ} \mathrm{C}$, and then a $3^{\circ} \mathrm{C} / \mathrm{s}$ ramp to $250^{\circ} \mathrm{C}$ with a $15 \mathrm{~s}$ hold. The temperature of the detector was $260^{\circ} \mathrm{C}$, and each sample was analyzed five times.

\section{Volatile Compound Analysis}

The volatile compound profiles of the CRW samples were analyzed using the headspace solid-phase microextraction (HS-SPME)/gas chromatography-mass spectrometry (GC-MS) approach (Yu et al., 2019b). Briefly, $5 \mathrm{~mL}$ of each CRW sample was added to $20 \mu \mathrm{L}$ of internal standard (2-octanol, $410 \mathrm{mg} / \mathrm{L}$ ) in a $15-\mathrm{mL}$ headspace glass vial. A fiber $(50 \mu \mathrm{m} \mathrm{DVB} / \mathrm{CAR} / \mathrm{PDMS}$, Supelco Inc., Bellefonte, Pennsylvania, United States) was exposed to the headspace of the glass vial for $50 \mathrm{~min}$ at $50^{\circ} \mathrm{C}$.

An Agilent 7890 GC instrument was coupled to a 5973C MS detector (Santa Clara, CA, United States). A capillary HP-Innowax column from Agilent Technologies $(60 \mathrm{~m} \times 0.25 \mathrm{~mm} \times 0.25 \mu \mathrm{m})$ was used to perform the chromatographic separation. After extraction, the fiber was immediately introduced into the GC instrument, and desorption was performed at $250^{\circ} \mathrm{C}$ for $5 \mathrm{~min}$. The temperature changes were as follows: the initial temperature was maintained at $40^{\circ} \mathrm{C}$, increased to $120^{\circ} \mathrm{C}$ at a rate of $3^{\circ} \mathrm{C} / \mathrm{min}$, held for $5 \mathrm{~min}$, and then increased to $200^{\circ} \mathrm{C}$ at a rate of $3^{\circ} \mathrm{C} / \mathrm{min}$. Helium was used as the carrier gas at a flow rate of $1 \mathrm{~mL} / \mathrm{min}$. The transfer line temperature was $250^{\circ} \mathrm{C}$. The mass spectrometers were operated in the electron ionization mode at $70 \mathrm{eV}$, with a scan range of $\mathrm{m} / \mathrm{z} 30-450$.

The compounds were identified by comparing their retention indices (RIs) with those reported in the literature and matching their MS spectra with those in the NIST 11 database. The RIs were determined in relation to those of the $\mathrm{C}_{5}-\mathrm{C}_{30}$ alkane standards (Sigma-Aldrich, St. Louis, MO, United States). To evaluate the sensory contributions of the compounds to the flavor of the CRW samples, their odor activity values (OAVs) were obtained, given by the ratio of the compound concentration in the sample to the threshold concentration in water (Van Gemert, 2003).

\section{Statistical Analysis}

XLSTAT version 7.5 (Addinsoft, New York, NY, United States) was used to analyze the GC-MS data. Design Expert software (version 9, Stat-Ease Inc., Minneapolis, MN, United States) was used for the regression and graphical analysis of the experimental data. The optimal values of the four protectants were calculated 

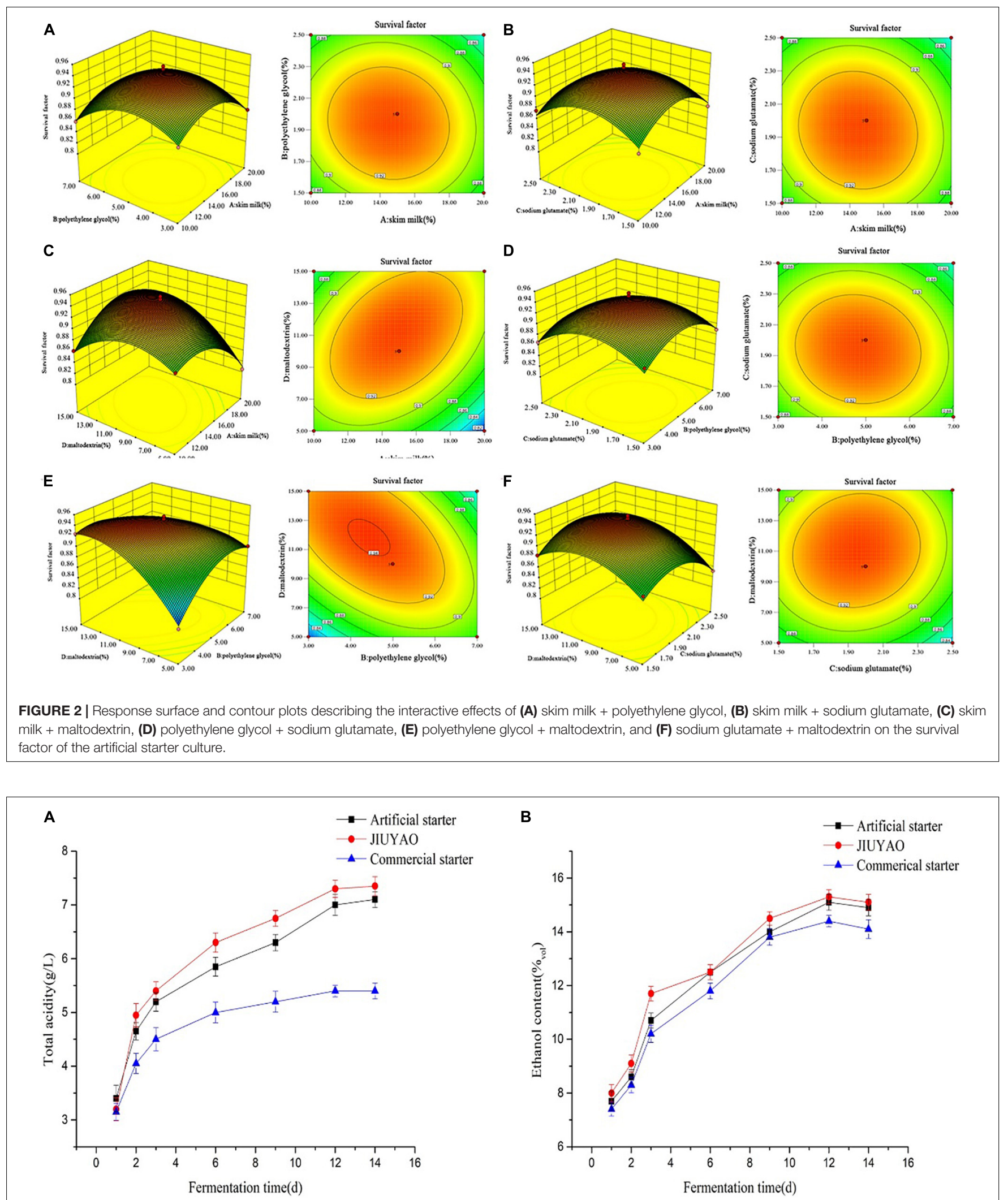

FIGURE 3 | Fermentation performance results of the three Chinese rice wine samples fermented with three different starter cultures. (A) Changes in the total acid contents during the fermentation process. (B) Changes in the ethanol contents during the fermentation process. 


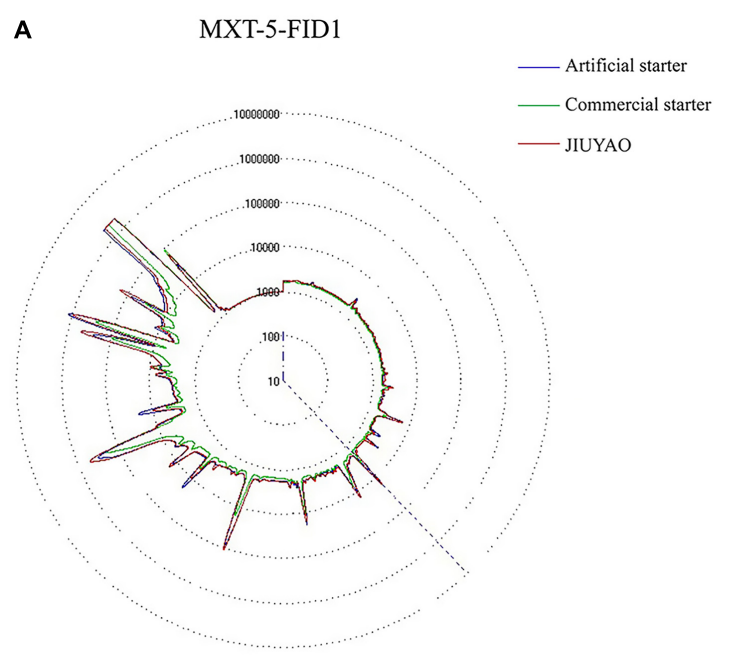

B

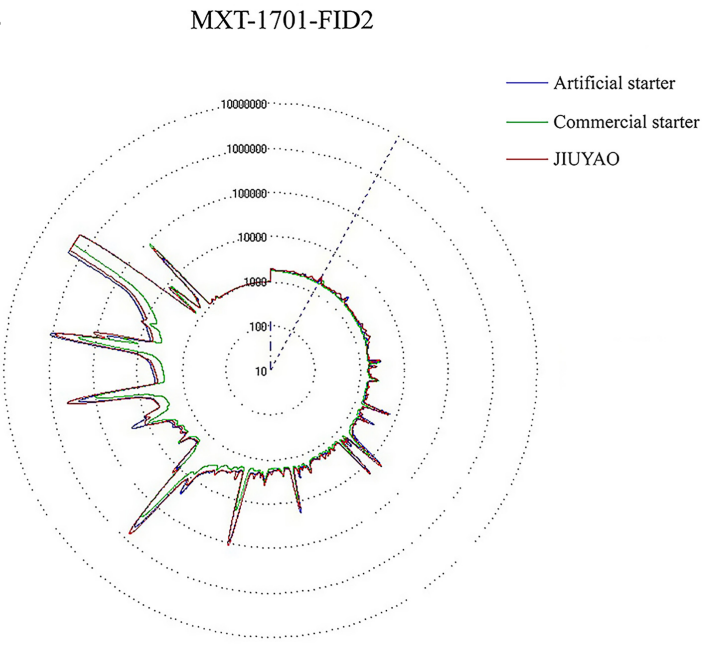

FIGURE 4 | Flavor profile radar chart of three Chinese rice wine samples brewed with three different starter cultures. (A) MXT-5-FID and (B) MXT-1701-FID.

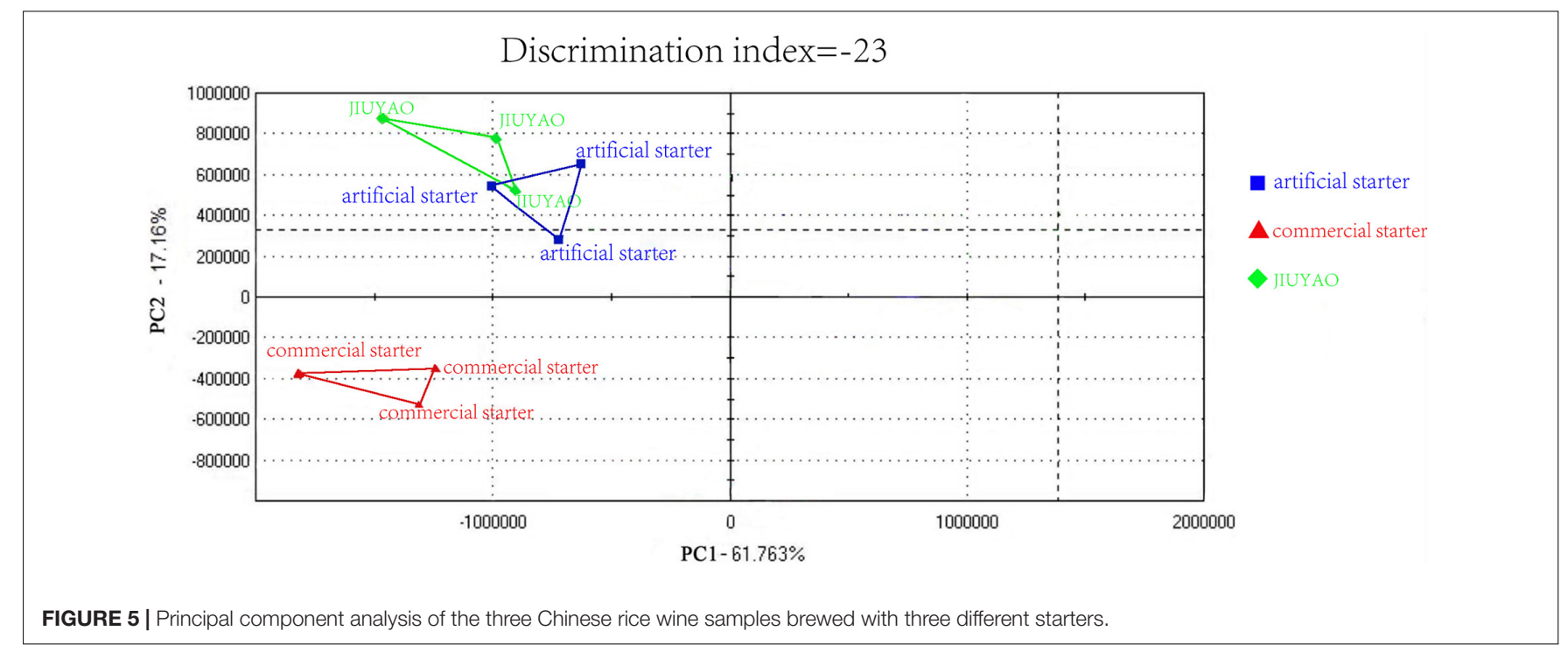

using response surface methodology. The regression equations of the models were evaluated using the $F$-test for the analysis of variance. A principal component analysis was performed using WinMuster version 1.6.2 (Alpha M.O.S., Toulouse, France). Origin version 9.0 (Origin Lab Inc., Hampton, MS, United States) and SPSS version 19.0 (SPSS Inc., Chicago, IL, United States) were used for further data analysis.

\section{RESULTS AND DISCUSSION}

\section{Effects of Lyoprotectants on the Survival Factor of the Artificial Starter After Freeze-Drying}

Lyophilization has several limitations, such as the formation of ice crystals, altered permeability of the cell membrane, and denaturation and inactivation of sensitive proteins (Yang et al., 2012; Lbg et al., 2020). The role of a lyoprotectant is to prevent these adverse effects. Skim milk, polyethylene glycol, sodium glutamate, and maltodextrin were selected as the protective agents for the artificial starter culture prepared in this study. The results of single-factor experiments indicated that with $15 \%$ skim milk, $5 \%$ polyethylene glycol, $2 \%$ sodium glutamate, or $10 \%$ maltodextrin as individual protective agents during freeze-drying, the maximum survival rate of the artificial starter culture was $85.55,80.94,83.83$, or $85.01 \%$, respectively (Figure 1). The survival factor of the artificial starter gradually improved with increasing concentrations of the protective agents, but after a certain concentration, the survival rate remained unchanged or slowly decreased. This phenomenon was in line with the results reported by Chen et al. (2019), who found that extremely high concentrations of protective agents accelerated the repolymerization of 
TABLE 3 | Relative contents of volatile compounds in the Chinese rice wine samples fermented by different starter cultures $(\mu \mathrm{g} / \mathrm{kg}, n=3)$, as identified by HS-SPME/GC-MS.

\begin{tabular}{|c|c|c|c|c|c|c|c|}
\hline \multirow[t]{2}{*}{ No } & \multirow[t]{2}{*}{ Compound $^{a}$} & \multirow[t]{2}{*}{ Identification ${ }^{b}$} & \multicolumn{2}{|c|}{$\mathbf{R I}$} & \multicolumn{3}{|c|}{ Concentration $(\mu \mathrm{g} / \mathrm{kg})$} \\
\hline & & & Calculate & Reference & $A^{c}$ & B & C \\
\hline 1 & Ethyl Acetate & $\mathrm{Rl}, \mathrm{MS}$ & 898 & 894 & $570.78 \pm 0.17 b^{d}$ & $613.63 \pm 0.96 c$ & $325.68 \pm 0.46 a$ \\
\hline 2 & Ethyl propionate & RI, MS & 969 & 964 & $25.92 \pm 0.07 b$ & $28.23 \pm 0.65 c$ & $11.86 \pm 0.16 a$ \\
\hline 3 & Isobutyl acetate & $\mathrm{Rl}, \mathrm{MS}$ & 1028 & 1029 & $11.59 \pm 0.32$ & $7.65 \pm 0.16$ & $-^{e}$ \\
\hline 4 & Ethyl butyrate & $\mathrm{RI}, \mathrm{MS}$ & 1031 & 1039 & $81.71 \pm 0.08 c$ & $63.16 \pm 0.28 b$ & $45.29 \pm 0.15 a$ \\
\hline 5 & Propanoic acid, pentyl ester & MS & 1064 & - & $20.44 \pm 0.64$ & - & - \\
\hline 6 & Isobutanol & RI, MS & 1086 & 1092 & $377.91 \pm 0.26 c$ & $161.82 \pm 0.48 b$ & $98.62 \pm 0.12 a$ \\
\hline 7 & Isoamyl acetate & $\mathrm{RI}, \mathrm{MS}$ & 1119 & 1126 & $464.08 \pm 0.15$ & $116.15 \pm 0.71$ & - \\
\hline 8 & 1-Butanol, 3- methyl-, acetate & $\mathrm{RI}, \mathrm{MS}$ & 1135 & 1129 & $416.49 \pm 0.15 b$ & $596.09 \pm 0.61 c$ & $348.98 \pm 0.54 a$ \\
\hline 9 & Pentanoic acid, ethyl ester & $\mathrm{RI}, \mathrm{MS}$ & 1140 & 1142 & $8.78 \pm 0.41$ & - & $62.38 \pm 0.48$ \\
\hline 10 & isopentanol & $\mathrm{RI}, \mathrm{MS}$ & 1200 & 1206 & $4459.53 \pm 10.14$ & $860.82 \pm 2.14$ & - \\
\hline 11 & 2-methylbutanol & $\mathrm{RI}, \mathrm{MS}$ & 1205 & 1208 & $3527.85 \pm 0.16$ & $338.94 \pm 0.12$ & - \\
\hline 12 & 2-Pentylfuran & $\mathrm{RI}, \mathrm{MS}$ & 1234 & 1235 & $26.57 \pm 0.54$ & $4.62 \pm 0.01$ & - \\
\hline 13 & ethyl hexanoate & $\mathrm{RI}, \mathrm{MS}$ & 1238 & 1241 & $185.64 \pm 0.15 a$ & $245.98 \pm 0.77 b$ & $556.31 \pm 0.84 c$ \\
\hline 14 & Acetic acid, hexyl ester & $\mathrm{RI}, \mathrm{MS}$ & 1270 & 1276 & $14.93 \pm 0.18$ & - & - \\
\hline 15 & Ethyl heptanoate & RI, MS & 1332 & 1332 & $18.24 \pm 0.20$ & $3.41 \pm 0.02$ & - \\
\hline 16 & 1-Hexanol & $\mathrm{RI}, \mathrm{MS}$ & 1365 & 1361 & $73.26 \pm 0.33$ & $1.8 \pm 0.08$ & - \\
\hline 17 & 1-Propanol, 3-ethoxy- & RI, MS & 1358 & 1359 & $65.46 \pm 0.34$ & $5.06 \pm 0.84$ & - \\
\hline 18 & 2-Nonanone & $\mathrm{RI}, \mathrm{MS}$ & 1395 & 1398 & $4.65 \pm 0.22$ & - & - \\
\hline 19 & acetic acid & $\mathrm{RI}, \mathrm{MS}$ & 1428 & 1427 & $82.83 \pm 0.15$ & - & $215.89 \pm 0.61$ \\
\hline 20 & ethyl caprylate & $\mathrm{RI}, \mathrm{MS}$ & 1436 & 1441 & $987.58 \pm 0.09$ & $1441.45 \pm 1.16$ & - \\
\hline 21 & 1-Octen-3-ol & $\mathrm{RI}, \mathrm{MS}$ & 1453 & 1456 & $13.53 \pm 0.25$ & $29.8 \pm 0.21$ & - \\
\hline 22 & furfural & $\mathrm{RI}, \mathrm{MS}$ & 1482 & 1482 & $10.42 \pm 0.15 a$ & $16.08 \pm 0.12 c$ & $15.56 \pm 0.51 b$ \\
\hline 23 & 1-Hexanol, 2-ethyl- & RI, MS & 1502 & 1484 & $12.15 \pm 0.61$ & - & - \\
\hline 24 & $\begin{array}{c}\text { Butanoic acid, 3- hydroxy-, } \\
\text { ethyl ester }\end{array}$ & $\mathrm{RI}, \mathrm{MS}$ & 1516 & 1522 & $34.92 \pm 0.55$ & - & - \\
\hline 25 & Ethyl nonanoate & $\mathrm{RI}, \mathrm{MS}$ & 1540 & 1541 & $84.28 \pm 0.40$ & $20.73 \pm 0.05$ & - \\
\hline 26 & n-Caprylic acid isobutyl ester & $\mathrm{RI}, \mathrm{MS}$ & 1557 & 1561 & $7.59 \pm 0.56$ & $5.02 \pm 0.03$ & - \\
\hline 27 & Caryophyllene & $\mathrm{RI}, \mathrm{MS}$ & 1593 & 1594 & $24.56 \pm 0.27$ & $11.12 \pm 0.12$ & - \\
\hline 28 & Ethyl decanoate & $\mathrm{RI}, \mathrm{MS}$ & 1640 & 1643 & $1021.13 \pm 0.11 b$ & $1829.47 \pm 2.98 c$ & $96.07 \pm 0.05 a$ \\
\hline 29 & Butanoic acid & $\mathrm{RI}, \mathrm{MS}$ & 1655 & 1652 & $18.1 \pm 0.31$ & - & - \\
\hline 30 & $\begin{array}{c}\text { Octanoic acid, 3-methylbutyl } \\
\text { ester }\end{array}$ & RI, MS & 1665 & 1670 & - & $15.71 \pm 0.17$ & - \\
\hline 31 & $\begin{array}{c}\text { Hexanoic acid, 3- hydroxy-, } \\
\text { ethyl ester }\end{array}$ & MS & 1673 & 1673 & $12.32 \pm 0.52$ & - & - \\
\hline 32 & Acetophenone & RI, MS & 1695 & 1693 & $68.63 \pm 0.29$ & - & $45.94 \pm 0.14$ \\
\hline 33 & 1-Decanol & $\mathrm{RI}, \mathrm{MS}$ & 1743 & 1748 & $61.47 \pm 0.13$ & $7.96 \pm 0.23$ & - \\
\hline 34 & Phenethyl acetate & $\mathrm{Rl}, \mathrm{MS}$ & 1800 & 1825 & $560.16 \pm 0.30$ & $351.1 \pm 0.24$ & - \\
\hline 35 & Ethyl laurate & $\mathrm{RI}, \mathrm{MS}$ & 1835 & 1835 & $197.88 \pm 0.10 b$ & $161.24 \pm 0.14 a$ & $536.96 \pm 0.11 c$ \\
\hline 36 & $\begin{array}{l}\text { Pentadecanoic acid, } \\
\text { 3-methylbutyl ester }\end{array}$ & RI, MS & 1862 & 1863 & $50.11 \pm 0.53$ & $8.19 \pm 0.06$ & - \\
\hline 37 & Phenylethyl Alcohol & $\mathrm{Rl}, \mathrm{MS}$ & 1936 & 1935 & $6686.56 \pm 0.06 b$ & $12940.7 \pm 5.17 c$ & $1584.71 \pm 0.96 a$ \\
\hline 38 & Ethyl Oleate & $\mathrm{RI}, \mathrm{MS}$ & 1988 & 1986 & $1230.5 \pm 0.36 c$ & $58.95 \pm 0.15 a$ & $629.12 \pm 21.28 b$ \\
\hline 39 & Phenol, 4-ethyl-2-methoxy- & $\mathrm{RI}, \mathrm{MS}$ & 2030 & 2032 & $90.28 \pm 0.24$ & $7.16 \pm 0.11$ & - \\
\hline 40 & Octanoic acid & RI, MS & 2040 & 2039 & $171.5 \pm 0.44$ & $28.28 \pm 0.05$ & - \\
\hline 41 & Tetradecanoic acid, ethyl ester & $\mathrm{RI}, \mathrm{MS}$ & 2053 & 2043 & $179.69 \pm 0.43 b$ & $68.65 \pm 0.51 a$ & $238.36 \pm 1.30 c$ \\
\hline 42 & Nonanoic acid & RI, MS & 2175 & 2169 & $45.58 \pm 0.39$ & - & - \\
\hline 43 & 2-Methoxy-4-vinylphenol & $\mathrm{RI}, \mathrm{MS}$ & 2196 & 2194 & $167.85 \pm 0.57$ & - & - \\
\hline 44 & Phenol, 4-ethyl- & $\mathrm{RI}, \mathrm{MS}$ & 2200 & 2202 & $115.39 \pm 0.38 c$ & $100.48 \pm 0.14 b$ & $54.89 \pm 0.19 a$ \\
\hline 45 & Hexadecanoic acid, ethyl ester & $\mathrm{RI}, \mathrm{MS}$ & 2243 & 2243 & - & $964.73 \pm 0.56$ & $7756.55 \pm 29.48$ \\
\hline 46 & Benzofuran, 2,3-dihydro- & MS & 2246 & - & $89.197 \pm 0.48$ & $6.9 \pm 0.47$ & - \\
\hline 47 & Ethyl 9-hexadecenoate & $\mathrm{RI}, \mathrm{MS}$ & 2272 & 2267 & $53.46 \pm 0.60$ & $20.44 \pm 0.58$ & - \\
\hline
\end{tabular}


TABLE 3 | Continued

\begin{tabular}{|c|c|c|c|c|c|c|c|}
\hline \multirow[t]{2}{*}{ No } & \multirow[t]{2}{*}{ Compound $^{\mathrm{a}}$} & \multirow[t]{2}{*}{ Identification $^{\mathrm{b}}$} & \multicolumn{2}{|c|}{$\mathbf{R I}$} & \multicolumn{3}{|c|}{ Concentration $(\mu \mathrm{g} / \mathrm{kg})$} \\
\hline & & & Calculate & Reference & $A^{c}$ & B & C \\
\hline 48 & n-Decanoic acid & $\mathrm{RI}, \mathrm{MS}$ & 2276 & 2275 & $92.57 \pm 0.19 b$ & $115.34 \pm 0.29 c$ & $53.68 \pm 0.10 a$ \\
\hline 49 & $\begin{array}{c}\text { Phenol, } \\
\text { 2,4-bis(1,1-dimethylethyl)- }\end{array}$ & $\mathrm{RI}, \mathrm{MS}$ & 2320 & 2321 & $72.43 \pm 0.28$ & $13.09 \pm 0.12$ & - \\
\hline 50 & Octadecanoic acid, ethyl ester & $\mathrm{RI}, \mathrm{MS}$ & 2450 & 2455 & $205.45 \pm 0.35 b$ & $33.51 \pm 0.28 a$ & $597.01 \pm 5.47 \mathrm{c}$ \\
\hline 51 & Linoleic acid ethyl ester & RI, MS & 2514 & 2521 & - & $31.57 \pm 0.17$ & $589.58 \pm 14.39$ \\
\hline
\end{tabular}

a Aroma compounds detected in the CRW samples.

${ }^{b}$ Method of identification: MS, mass spectrum comparison using NIST11.L library; RI, retention index in agreement with literature value.

${ }^{C} A, B, C$ are CRW samples fermented with JIUYAO, artificial starter and commercial starter.

'Values with different letters $(\mathrm{a}-\mathrm{C})$ in a row are significantly different using Duncan's multiple comparison tests $(p<0.05)$.

e Not detected in sample.

proteins in the cells, resulting in poor survival of the artificial starter.

\section{Predictive Modeling for the Concentration of Protective Agents}

To further improve the survival factor of the microbes in the artificial starter, a predictive model was established for the optimal protectant combination comprising skim milk, polyethylene glycol, sodium glutamate, and maltodextrin. Based on the combination of the Box-Behnken design and the response surface method, an empirical quadratic model was selected to establish the correlation between the independent variables and the response of the survival factor, as follows:

$$
\begin{aligned}
\mathrm{Y}= & -0.393+0.036 \mathrm{~A}+0.132 \mathrm{~B}+0.617 \mathrm{C}+0.028 \mathrm{D} \\
& +3.25 \times 10^{-4} \mathrm{AB}-2.40 \times 10^{-3} \mathrm{AC}+1.15 \\
& \times 10^{-3} \mathrm{AD}-5.75 \times 10^{-3} \mathrm{BC}-3.725 \times 10^{-3} \mathrm{BD} \\
& +2.6 \times 10^{-3} \mathrm{CD}-1.526 \times 10^{-3} \mathrm{~A}^{2}-9.131 \times 10^{-3} \mathrm{~B}^{2} \\
& -0.149 \times 10^{-3} \mathrm{C}^{2}-1.446 \times 10^{-3} \mathrm{D}^{2}
\end{aligned}
$$

The $F$-value of the model was 35.22 , indicating that this model was significant. The $p$-values of the lack of fit $(p=0.3293)$ and the model $(p<0.0001)$ (Table 2$)$ indicated that the actual corresponding survival factor values exhibited a good fit with this model.

The effects of the tested factors on the survival factor were visualized in the response surfaces (Figure 2). The interaction terms of the concentrations of skim milk, polyethylene glycol, sodium glutamate, and maltodextrin demonstrated statistical significance $(p<0.0001)$. The survival factor peaked at 0.942 when the concentrations of skim milk, polyethylene glycol, sodium glutamate, and maltodextrin were $15.09,4.45,1.96$, and $11.81 \%$, respectively. Using this combination, the survival factor increased significantly by approximately $10 \%$ relative to the use of single protective agents before optimization. This suggests that the established predictive model could effectively predict the survival factor. Hence, an artificial starter was established with the optimal protectant combination of $15.09 \%$ skim milk, $4.45 \%$ polyethylene glycol, $1.96 \%$ sodium glutamate, and $11.81 \%$ maltodextrin. To further verify the predictive value of the response surface optimization, three repeat experiments were performed using the optimal protectant combination. The average value of the survival factor of the artificial starter was 0.931 , and the fitting rate of the predicted value was $98.83 \%$, indicating that the predicted value and the actual value had a good fit. Together, these data suggest that the determined optimal protectant composition can significantly improve the survival factor of the artificial starter.

\section{Fermentation Activity Analysis of the CRW Samples Brewed With Different Starters}

We compared the fermentation activities of the three CRW samples, each brewed with either traditional JIUYAO, the commercial starter, or the artificial starter prepared with the optimal lyoprotectant composition obtained by the response surface method. Saccharification capacity is a key factor that significantly influences wine fermentation (Yao et al., 2018). JIUYAO showed the highest saccharification capacity at $290 \pm 3.2 \mathrm{mg} / \mathrm{g} \mathrm{h}$, followed by the artificial starter $(275 \pm 5.3 \mathrm{mg} / \mathrm{g} \mathrm{h})$, while the commercial starter showed the lowest capacity $(200 \pm 4.6 \mathrm{mg} / \mathrm{g} \mathrm{h})$. The fermentation activity (ethanol and acid content) of the three CRW samples during fermentation is shown in Figure 3. As CRW is mostly fermented in an open environment, rapid growth of the yeast strains in the starter is required to produce ethanol at the initial stage to inhibit bacterial overgrowth and avoid spoilage (Wu et al., 2015; Yang et al., 2018). The changes in ethanol content during fermentation were similar across the three samples, suggesting comparable ethanol production capacities of all three starter cultures. The total acidity of the wines brewed with JIUYAO and the artificial starter were similar, and were higher than that of the wine brewed with the commercial starter throughout the whole fermentation process. These results suggest that the fermentation activity of the CRW sample fermented with the artificial starter was comparable to that of the sample fermented with JIUYAO, but more vigorous than that of the sample fermented with the commercial starter.

\section{Electronic Nose Measurements}

Combining the high-efficiency separation ability of GC and the biological simulation of the sense of smell, the electronic nose can provide a comprehensive aroma profile of volatile flavor compounds (Wu et al., 2012). As shown in Figure 4, the 
flavor profile radar chart of the CRW samples brewed with the three different starters displays the data intuitively. The overall aroma peak appearance of the samples brewed with JIUYAO and the artificial starter is relatively similar, whereas the peak areas are reduced at multiple positions for the sample fermented with the commercial starter, indicating an overall reduction in aroma intensity. To enable better data discrimination between the three samples, a principal component analysis was performed to identify patterns associated with their individual components (Figure 5). The principal components of the CRW samples fermented with the artificial starter and JIUYAO were closer and overlapped in the same quadrant, suggesting similar aroma profiles, whereas those of the CRW sample brewed with the commercial starter appeared in a different quadrant. These results indicate similarities in the aroma profiles between the CRW samples brewed with the artificial starter and JIUYAO, but some differences in the CRW sample brewed with the commercial starter. These differences may be ascribed to the microbial species composition. Five core species isolated from JIUYAO formed the functional microbial species of the artificial starter culture, whereas the commercial starter contained only two functional microbial species, namely $S$. cerevisiae and R. oryzae.

\section{Volatile Flavor Compounds of the CRW Samples Fermented With Different Starters}

In total, 51 flavor compounds, including 27 esters, 8 alcohols, 1 aldehyde, 4 phenols, 2 ketones, and 5 acids, were detected in the three CRW samples by HS-SPME/GC-MS (Table 3). A total of 50,39 , and 20 aroma compounds were identified in the CRW

TABLE 4 | OAVs of volatile compounds detected in the three Chinese rice wine samples fermented with three different starter cultures.

\begin{tabular}{|c|c|c|c|c|c|}
\hline No & Compounds & $\mathbf{A}^{\mathrm{a}}$ & B & C & $\begin{array}{c}\text { Threshold }^{\mathrm{c}} \\
(\mu \mathrm{g} / \mathrm{kg})\end{array}$ \\
\hline 1 & Phenylethyl alcohol & 111 & 216 & 26 & 60 \\
\hline 2 & Ethyl caprylate & 5 & 7 & $-b$ & 200 \\
\hline 3 & Ethyldecanoate & 2 & 3 & $<1$ & 530 \\
\hline 4 & Isoamyl acetate & 208 & 298 & 174 & 2 \\
\hline 5 & 1-octen-3-ol & 5 & 11 & - & 2.7 \\
\hline 6 & Ethyl hexanoate & $<1$ & $<1$ & 1 & 530 \\
\hline 7 & Ethyl butyrate & 1 & 1 & $<1$ & 59 \\
\hline 8 & Ethyl propionate & 1 & 1 & $<1$ & 29 \\
\hline 9 & Ethyl acetate & 114 & 123 & 65 & 5 \\
\hline 10 & Furfural & 1 & 2 & - & 8 \\
\hline 11 & Ethyl laurate & $<1$ & $<1$ & 1 & 500 \\
\hline 12 & Isoamyl alcohol & 782 & 689 & - & 6.1 \\
\hline 13 & Isobutanol & 12 & 5 & - & 33 \\
\hline 14 & n-Decanoic acid & 1 & 2 & $<1$ & 70 \\
\hline
\end{tabular}

${ }^{a} A, B, C$ are CRW samples fermented with JIUYAO, the artificial starter and the commercial starter.

${ }^{b}$ The OAV was not calculated in the sample.

cThe detection threshold was drawn from the literature (Van Gemert, 2003). samples fermented with JIUYAO, the artificial starter, and the commercial starter, respectively.

Esters are the most important and common volatile aroma compounds that impart floral and fruity sensory properties to wine (Huang L. et al., 2018), and can be synthesized by yeast and other microorganisms during fermentation (Comuzzo et al., 2006). In the present study, esters formed the largest group of flavor compounds, with 26 and 13 ester compounds detected in the CRW samples fermented with JIUYAO and the artificial starter, respectively. The ester content of the wine fermented with the artificial starter $(6685.06 \mu \mathrm{g} / \mathrm{kg})$ was comparable to that of the wine fermented with JIUYAO $(7076.73 \mu \mathrm{g} / \mathrm{kg})$, but significantly $(p<0.05)$ higher than that of the wine fermented with the commercial starter $(4794.15 \mu \mathrm{g} / \mathrm{kg})$. Notably, isobutyl acetate, n-caprylic acid isobutyl ester, ethyl heptanoate, ethyl nonanoate, phenethyl acetate, and ethyl caprylate were the only esters identified in the CRW samples brewed with JIUYAO and the artificial starter. Thus, these ester compounds may be significantly correlated with the core microbial species used in the artificial starter.

Alcohols formed the second largest category of flavor compounds in the CRW samples, and are known to be the key aroma components of wine, especially brewed rice wine (Wang et al., 2014). Alcohols are produced through the metabolism of sugars and the decarboxylation and dehydrogenation of amino acids (Hernandez-Orte et al., 2008). Nine, eight, and two alcohol compounds were detected in the CRW samples fermented with JIUYAO, the artificial starter, and the commercial starter, respectively. Only two alcohols, namely isobutanol and phenylethyl alcohol, were identified in the wine fermented with the commercial starter. The alcohol content of the CRW samples fermented with JIUYAO and the artificial starter was 15,212.26 and $14,341.84 \mu \mathrm{g} / \mathrm{kg}$, respectively, but that of the sample fermented with the commercial starter was only $1683.33 \mu \mathrm{g} / \mathrm{kg}$. These results indicate that the core microorganisms used in the artificial starter could produce considerably more alcohol compounds during CRW fermentation.

To assess the complex olfactory effects of the different aroma compounds, individual OAVs were calculated (Bavcar et al., 2011). As shown in Table 4, 14 volatile compounds with OAVs $\geq 1$ were identified in the three CRW samples, including 8 esters, 4 alcohols, 1 acid, and 1 aldehyde. Twelve aroma compounds with OAVs $\geq 1$ were found in the CRW samples fermented with JIUYAO and the artificial starter, but only five were identified in the sample fermented with the commercial starter. Among these compounds, ethyl caprylate, 1-octen-3-ol, ethyl decanoate, ethyl butyrate, furfural, isoamyl alcohol, ethyl propionate, isobutanol, and n-decanoic acid were detected only in the samples brewed with JIUYAO and the artificial starter. These compounds contribute to the pleasant aroma profile of CRW due to their desirable aroma and low odor threshold (Yu et al., 2019a). For example, ethyl butyrate imparts an apple-like aroma to rice wine. Isoamyl alcohol is a powerful aroma agent with a banana flavor, which can improve the taste of wine by reducing the bittertasting amino acids (leucine) (Chen Z. et al., 2015). These results, together with those of the electronic nose analysis, indicate that the flavor profile-especially the key aroma compounds-of the 
CRW fermented with the artificial starter is similar to that of the CRW fermented with JIUYAO. Thus, the artificial starter is a potential substitute to JIUYAO to aid the industrial production of high-quality CRW with a stable flavor profile.

\section{CONCLUSION}

An artificial starter was prepared for high-efficiency industrial CRW production, and the fermentation activities and flavor profiles of CRW samples fermented with JIUYAO, a commercial starter culture, and our artificial starter culture were compared. The optimal lyoprotectant combination was determined as $15.09 \%$ skim milk, $4.45 \%$ polyethylene glycol, $1.96 \%$ sodium glutamate, and $11.81 \%$ maltodextrin, using the response surface optimization method. These three different starters had equivalent fermentation activity in terms of the alcohol content of the fermented CRW samples. The sample brewed with the artificial starter showed similar acid content and volatile compound profiles to those of the sample brewed with JIUYAO. Although the aroma compound content of the CRW sample fermented with the artificial starter was lower than that of the sample fermented with JIUYAO, the same main aroma compounds, such as isoamyl acetate, ethyl acetate, phenyl alcohol, and isoamyl alcohol (OAV $\geq 1$ ), were found in both and contributed highly to the flavor of the CRW. Our artificial starter culture is a promising substitute to traditional JIUYAO to aid the industrial production of high-quality CRW with a stable flavor profile. Further studies should be devoted to in-depth analysis of the associations between the core microbes and flavor substances to enhance the activity and stability of the artificial starter during batch production of CRW.

\section{REFERENCES}

Abadias, M., Benabarre, A., Teixidó, N., Usall, J., and ViñAs, I. (2001). Effect of freeze drying and protectants on viability of the biocontrol yeast Candida sake. Int. J. Food Microbiol. 65, 173-182. doi: 10.1016/S0168-1605(00)00513-4

Ale, C. E., Otero, M. C., and Pasteris, S. E. (2015). Freeze-drying of wine yeasts and oenococcus oeni and selection of the inoculation conditions after storage. J. Bioprocess. Biotech. 5:248.

Bavcar, D., Cesnik, H. B., Cus, F., and Kosmerl, T. (2011). The influence of skin contact during alcoholic fermentation on the aroma composition of Ribolla Gialla and Malvasia Istriana Vitis vinifera (L.) grape wines. Int. J. Food Sci. Tech. 46, 1801-1808. doi: 10.1111/j.1365-2621.2011.02679.x

Cerrutti, P., Huergo, M., Galvagno, M., Schebor, C., and Buera, M. (2000). Commercial baker's yeast stability as affected by intracellular content of trehalose, dehydration procedure and the physical properties of external matrices. Appl. Microbiol. Biot. 54, 575-580. doi: 10.1007/s002530000428

Chen, C., Liu, Y., Tian, H., Ai, L., and Yu, H. (2020). Metagenomic analysis reveals the impact of JIUYAO microbial diversity on fermentation and the volatile profile of Shaoxing-jiu. Food Microbiol. 86, 103321-103326. doi: 10.1016/j.fm. 2019.103326

Chen, H., Chen, S., Li, C., and Shu, G. (2015). Response surface optimization of lyoprotectant for lactobacillus bulgaricus during vacuum freeze-drying. Prep. Biochem. Biotech. 45, 463-475. doi: 10.1080/10826068.2014.923451

Chen, H., Tian, M., Chen, L., Cui, X., Meng, J., and Shu, G. (2019). Optimization of composite cryoprotectant for freeze-drying Bifidobacterium bifidum BB01 by

\section{DATA AVAILABILITY STATEMENT}

The original contributions presented in the study are included in the article/supplementary material, further inquiries can be directed to the corresponding author/s.

\section{AUTHOR CONTRIBUTIONS}

CC wrote the manuscript and performed the statistical analyses. ZL performed the statistical analyses and flash GC electronic nose detection. WZ compared the fermentation activities between different samples. HT determined the flavor profiles of samples by gas chromatography-mass spectrometry. $\mathrm{JH}$ and HYua established the optimal protectant combination for lyophilization of the artificial starter. HYu designed the research. All authors contributed to the article and approved the submitted version.

\section{FUNDING}

This work was supported by the National Natural Science Foundation of China (32172336) and Capacity Project of Local Colleges and Universities of the Science and Technology Commission of Shanghai, China (21010504100).

\section{ACKNOWLEDGMENTS}

The authors are grateful to the Zhejiang Tapai Shaoxing Rice Wine Co., Ltd. for their generous supply of materials for this study.

response surface methodology. Artif. Cells Nanomed. Biotechnol. 47, 1559-1569. doi: 10.1080/21691401.2019.1603157

Chen, S., Xu, Y., and Qian, M. C. (2013). Aroma characterization of Chinese rice wine by gas chromatography-olfactometry, chemical quantitative analysis, and aroma reconstitution. J. Agric. Food Chem. 61, 11295-11302. doi: 10.1021/ jf4030536

Chen, Z., Jia, R., Liu, Z., Zhang, W., Chen, S., Rao, P., et al. (2015). Microbial community structure and dynamics during the traditional brewing of Fuzhou Hong Qu glutinous rice wine as determined by culture-dependent and cultureindependent techniques. Food Control 57, 216-224. doi: 10.1016/j.foodcont. 2015.03.054

Comuzzo, P., Tat, L., Tonizzo, A., and Battistutta, F. (2006). Yeast derivatives (extracts and autolysates) in winemaking: release of volatile compounds and effects on wine aroma volatility. Food Chem. 99, 217-230. doi: 10.1016/j. foodchem.2005.06.049

Coulibaly, W. H., N'guessan, K. F., Coulibaly, I., Cot, M., Rigou, P., and Djè, K. M. (2016). Influence of freeze-dried yeast starter cultures on volatile compounds of tchapalo, a traditional sorghum beer from cte d'ivoire. Beverages 2:35. doi: 10.3390/beverages2040035

Crowell, E. A., and Ough, C. S. (1979). A modified procedure for alcohol determination by dichromate oxidation. Am. J. Enol. Vitic. 30, 61-63.

Ferrer-Gallego, R., Hernández-Hierro, J., Rivas-Gonzalo, J. C., and EscribanoBailón, M. (2014). Sensory evaluation of bitterness and astringency subqualities of wine phenolic compounds: synergistic effect and modulation by aromas. Food Res. Int. 62, 1100-1107. doi: 10.1016/j.foodres.2014.05.049 
Hernandez-Orte, P., Cersosimo, M., Loscos, N., Cacho, J., Garcia-Moruno, E., and Ferreira, V. (2008). The development of varietal aroma from non-floral grapes by yeasts of different genera. Food Chem. 107, 1064-1077. doi: 10.1016/ j.foodchem.2007.09.032

Huang, L., Ma, Y., Tian, X., Li, J. M., Li, L. X., Tang, K., et al. (2018). Chemosensory characteristics of regional Vidal icewines from China and Canada. Food Chem. 261, 66-74. doi: 10.1016/j.foodchem.2018.04.021

Huang, Z. R., Guo, W. L., Zhou, W. B., Li, L., Xu, J. X., Hong, J. L., et al. (2019). Microbial communities and volatile metabolites in different traditional fermentation starters used for Hong Qu glutinous rice wine. Food Res. Int. 121, 593-603. doi: 10.1016/j.foodres.2018.12.024

Huang, Z. R., Hong, J. L., Xu, J. X., Li, L., Guo, W. L., Pan, Y. Y., et al. (2018). Exploring core functional microbiota responsible for the production of volatile flavour during the traditional brewing of Wuyi Hong Qu glutinous rice wine. Food Microbiol. 76, 487-496. doi: 10.1016/j.fm.2018.07.014

Hubálek, Z. (2003). Protectants used in the cryopreservation of microorganisms. Cryobiology 46, 205-229. doi: 10.1016/S0011-2240(03)00046-4

Jiao, A., Xu, X., and Jin, Z. (2017). Research progress on the brewing techniques of new-type rice wine. Food Chem. 215, 508-515. doi: 10.1016/j.foodchem.2016. 08.014

Lbg, A., Ahc, A., and Og, B. (2020). Storage stability and sourdough acidification kinetic of freeze-dried Lactobacillus curvatus N19 under optimized cryoprotectant formulation. Cryobiology 96, 122-129. doi: 10.1016/j. cryobiol.2020.07.007

Liu, S., Chen, Q., Zou, H., Yu, Y., and Zhang, S. (2019). A metagenomic analysis of the relationship between microorganisms and flavor development in Shaoxing mechanized huangjiu fermentation mashes. Int. J. Food Microbiol. 303, 9-18. doi: 10.1016/j.ijfoodmicro.2019.05.001

Liu, Z., Wang, Z., Lv, X., Zhu, X., and Li, N. (2018). Comparison study of the volatile profiles and microbial communities of Wuyi Qu and Gutian Qu, two major types of traditional fermentation starters of Hong Qu glutinous rice wine. Food Microbiol. 69, 105-115. doi: 10.1016/j.fm.2017.07.019

Pradelles, R., Vichi, S., Alexandre, H., and Chassagne, D. (2009). Influence of the drying processes of yeasts on their volatile phenol sorption capacity in model wine. Int. J. Food Microbiol. 135, 152-157. doi: 10.1016/j.ijfoodmicro.2009.07. 019

Qi, K., He, C., Wan, H., Man, H., and Wu, Y. (2017). Response surface optimization of lyoprotectant from amino acids and salts for bifidobacterium bifidum during vacuum freeze-drying. Acta Univ. Cibiniensis Ser. E Food Technol. 21, 3-10. doi: 10.1515/aucft-2017-0009

Van Gemert, L. (2003). Compilations of Odour Threshold Values in Air, Water and Other Media. Zeist: Oliemans, Punter \& Partners B.V.

Wang, P., Mao, J., Meng, X., Li, X., Liu, Y., and Feng, H. (2014). Changes in flavour characteristics and bacterial diversity during the traditional fermentation of Chinese rice wines from Shaoxing region. Food Control 44, 58-63. doi: 10.1016/ j.foodcont.2014.03.018

Wu, K. N., Tan, B. K., Howard, J. D., Conley, D. B., and Gottfried, J. A. (2012). Olfactory input is critical for sustaining odor quality codes in human orbitofrontal cortex. Nat. Neurosci. 15, 1313-1319. doi: 10.1038/nn. 3186

Wu, Z., Xu, E., Jie, L., Zhang, Y., Fang, W., Xu, X., et al. (2015). Monitoring of fermentation process parameters of Chinese rice wine using attenuated total reflectance mid-infrared spectroscopy. Food Control 50, 405-412. doi: 10.1016/ j.foodcont.2014.09.028

Xie, G. F., Li, W. J., Jian, L., Yu, C., Hua, F., Zou, H. J., et al. (2007). Isolation and identification of representative fungi from Shaoxing rice wine Wheat $\mathrm{Qu}$ using a polyphasic approach of culture-based and molecular-based methods. J. Inst. Brew. 113, 272-279. doi: 10.1002/j.2050-0416.2007.tb00287.x

Xu, E., Jie, L., Wu, Z., Li, H., and Jiao, A. (2015). Characterization of volatile flavor compounds in Chinese rice wine fermented from enzymatic extruded rice. J. Food Sci. 80, 1476-1489. doi: 10.1111/1750-3841.12935

Yang, C., Zhu, X., Fan, D., Mi, Y., Luo, Y., Hui, J., et al. (2012). Optimizing the chemical compositions of protective agents for freeze-drying bifidobacterium longum BIOMA 5920. Chin. J. Chem. Eng. 20, 930-936. doi: 10.1016/S10049541(12)60420-0

Yang, Y. J., Xia, Y. J., Lin, X. N., Wang, G. Q., Zhang, H., Xiong, Z. Q., et al. (2018). Improvement of flavor profiles in Chinese rice wine by creating fermenting yeast with superior ethanol tolerance and fermentation activity. Food Res. Int. 108, 83-92. doi: 10.1016/j.foodres.2018.03.036

Yao, L. D., Ju, X., James, T. Y., Qiu, J. Z., and Liu, X. Y. (2018). Relationship between saccharifying capacity and isolation sources for strains of the Rhizopus arrhizus complex. Mycoscience 59, 409-414. doi: 10.1016/j.myc.2018.02.011

Yu, H., Xie, T., Xie, J., Ai, L., and Tian, H. (2019b). Characterization of key aroma compounds in Chinese rice wine using gas chromatography-mass spectrometry and gas chromatography-olfactometry. Food Chem. 293, 8-14. doi: 10.1016/j. foodchem.2019.03.071

Yu, H., Xie, T., Qian, X., Ai, L., and Tian, H. (2019a). Characterization of the volatile profile of Chinese rice wine by comprehensive two-dimensional gas chromatography coupled to quadrupole mass spectrometry. J. Sci. Food Agr. 99, 5444-5456. doi: 10.1002/jsfa.9806

Zhao, G., and Zhang, G. (2010). Effect of protective agents, freezing temperature, rehydration media on viability of malolactic bacteria subjected to freeze-drying. J. Appl. Microbiol. 99, 333-338. doi: 10.1111/j.1365-2672.2005.02587.x

Zhao, X., Wang, Y., Cai, W., Yang, M., and Shan, C. (2020). High-throughput sequencing-based analysis of microbial diversity in rice wine koji from different areas. Curr. Microbiol. 77, 1-8. doi: 10.1007/s00284-020-01877-9

Conflict of Interest: The authors declare that the research was conducted in the absence of any commercial or financial relationships that could be construed as a potential conflict of interest.

Publisher's Note: All claims expressed in this article are solely those of the authors and do not necessarily represent those of their affiliated organizations, or those of the publisher, the editors and the reviewers. Any product that may be evaluated in this article, or claim that may be made by its manufacturer, is not guaranteed or endorsed by the publisher.

Copyright (C) 2021 Chen, Liu, Zhou, Tian, Huang, Yuan and Yu. This is an openaccess article distributed under the terms of the Creative Commons Attribution License (CC BY). The use, distribution or reproduction in other forums is permitted, provided the original author(s) and the copyright owner(s) are credited and that the original publication in this journal is cited, in accordance with accepted academic practice. No use, distribution or reproduction is permitted which does not comply with these terms. 